\title{
Resistance furnace temperature control based on prediction BP neural network
}

\author{
Yaowu Tang \\ College of Electrical Engineering, \\ Jilin Teachers' Institute of Engineering \&Technology \\ Changchun 130052 China \\ E-mail:158540915@QQ.COM
}

\begin{abstract}
For resistance furnace nonlinear, time-varying, hysteresis characteristics, the use of predictive control of advance and BP neural network has the ability to approximate any nonlinear function of the characteristics of the design of the neural network predictive controller resistance furnace temperature control. The simulation results show that this method enhances the tracking of the system, to have a good time delay compensation, accused parameter has a strong ability to adapt than traditional control methods precision, speed, excellent quality control. Keywords: resistance furnace; BP neural network; predictive control
\end{abstract}

\section{Introduction}

Resistance furnace with nonlinear, time-varying, multi-disturbance, hysteresis and other properties, using conventional means of mathematical modeling and classical control methods are difficult to produce the desired control its effects. In recent years, according to current and past information to predict the future output of predictive control algorithm is widely used in industrial control process, to enhance the tracking of the system; and BP neural network has the ability to approximate any nonlinear function, and the structures and algorithms simple and clear, able to accurately describe the dynamic process of uncertainty. Therefore, in order to overcome the deficiencies of traditional control algorithm, the BP neural networks and predictive control to design a combination of BP neural network predictive controller of resistance furnace temperature control, simulation results show that this method than the traditional method of temperature control accuracy high speed, can effectively overcome resistance furnace Delays and time variability, excellent quality control.

\section{the temperature control system works}

The actual temperature is detected by a thermocouple in the furnace, the temperature transmitter is converted to a voltage signal $0 \sim 5 \mathrm{~V}$, and after the acquisition and the computer compares the set temperature to obtain the rate of 
change of the temperature error and error, using BP neural network predictive control algorithm obtaining control output. The output to the input of the SCR regulator change the SCR conduction angle. Conduction angle is larger, the AC voltage supplied to the furnace at both ends of the resistor will be higher, the resistance furnace also increases input power, temperature rise; the other hand, the conduction angle decreases, the input power decreases resistance furnace, the furnace temperature drop; furnace temperature deviation is zero, the thyristor conduction angle to maintain a certain, constant power input resistance furnace. By controlling the thyristor conduction angle controlled resistance heater current, and thus the temperature of the controlled object, so that the actual temperature of the object toward a given change in temperature and eventually reaches a given temperature.

\section{the control system configured}

Resistance furnace BP neural network predictive control system consists of an object resistance furnace, furnace temperature controlled neural network forecasting model, feedback correction, temperature rolling optimization controller. The principle is shown in Figure 1.

Furnace temperature prediction model, used to approximate the actual object, which according to current and historical system input and output information to predict future furnace; feedback correction for correcting the output prediction model furnace, stove lose Out error, in order to improve the prediction accuracy of prediction model furnace; furnace rolling optimization controller role is to calculate the amount of the difference between optimal control reference trajectory based on predicted output of the system.

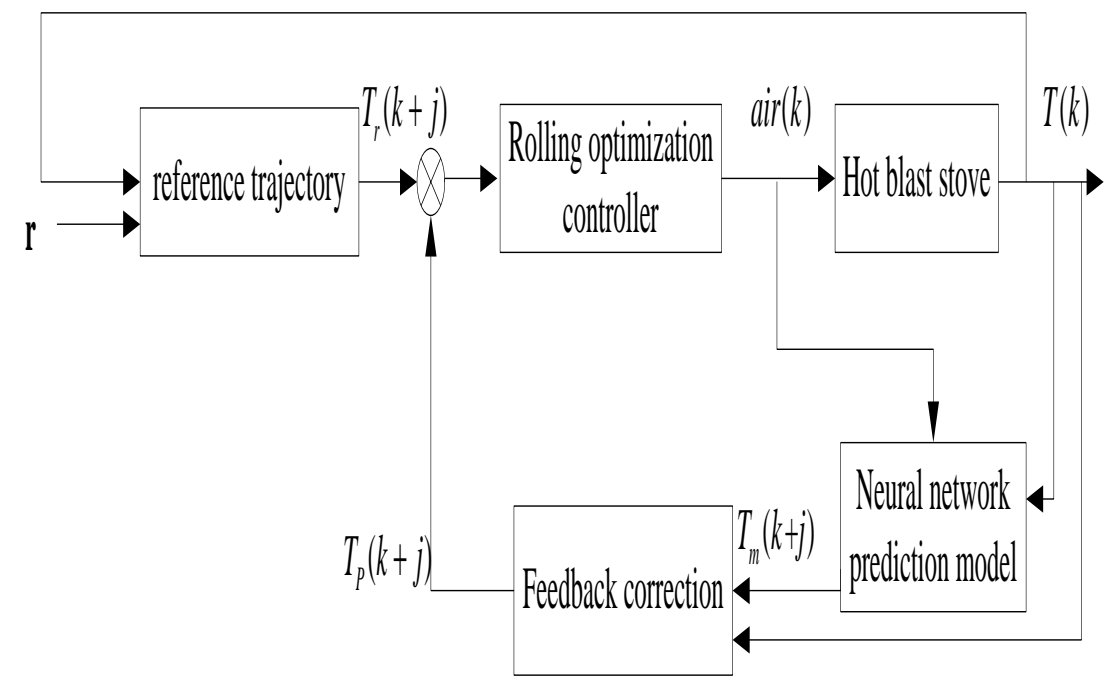

Fig.1 The principle diagram of the Resistance furnace temperature control 
system

\section{The establishment of theResistance furnace model} below.

The relationship between the blast volume and temperature of $\mathrm{T}$, as shown

$$
T_{m}(k)=f\left(T(k-1), \cdots, T\left(k-n_{a}\right), \operatorname{air}(k-1), \cdots, \operatorname{air}\left(k-n_{b}\right)\right)+\varepsilon(k)
$$

$T_{m}(k)$ is the predicted temperature of furnace temperature prediction model.

$T(k)$ is the temperature at time $\mathrm{k}$.

$\operatorname{air}(k)$ is $\mathrm{k}$ times of air supply.

$n_{a}$ is the system input,$n_{b}$ is the system output order.

$\varepsilon(k)$ is the white noise sequence.

$f(\cdot)$ is a nonlinear function. $f(\cdot) \in L^{2}\left(R^{n_{a}+n_{b}}\right)$.

Neural network to approximate continuous or discontinuous function. With three layers of BP neural network to construct the hot blast furnace temperature prediction model. Prediction model structural diagram is shown in figure 2. The model consists of an input layer, one hidden layer and one output layer. Implicit response function layer is generally nonlinear function, such as the Sigmoid function. 

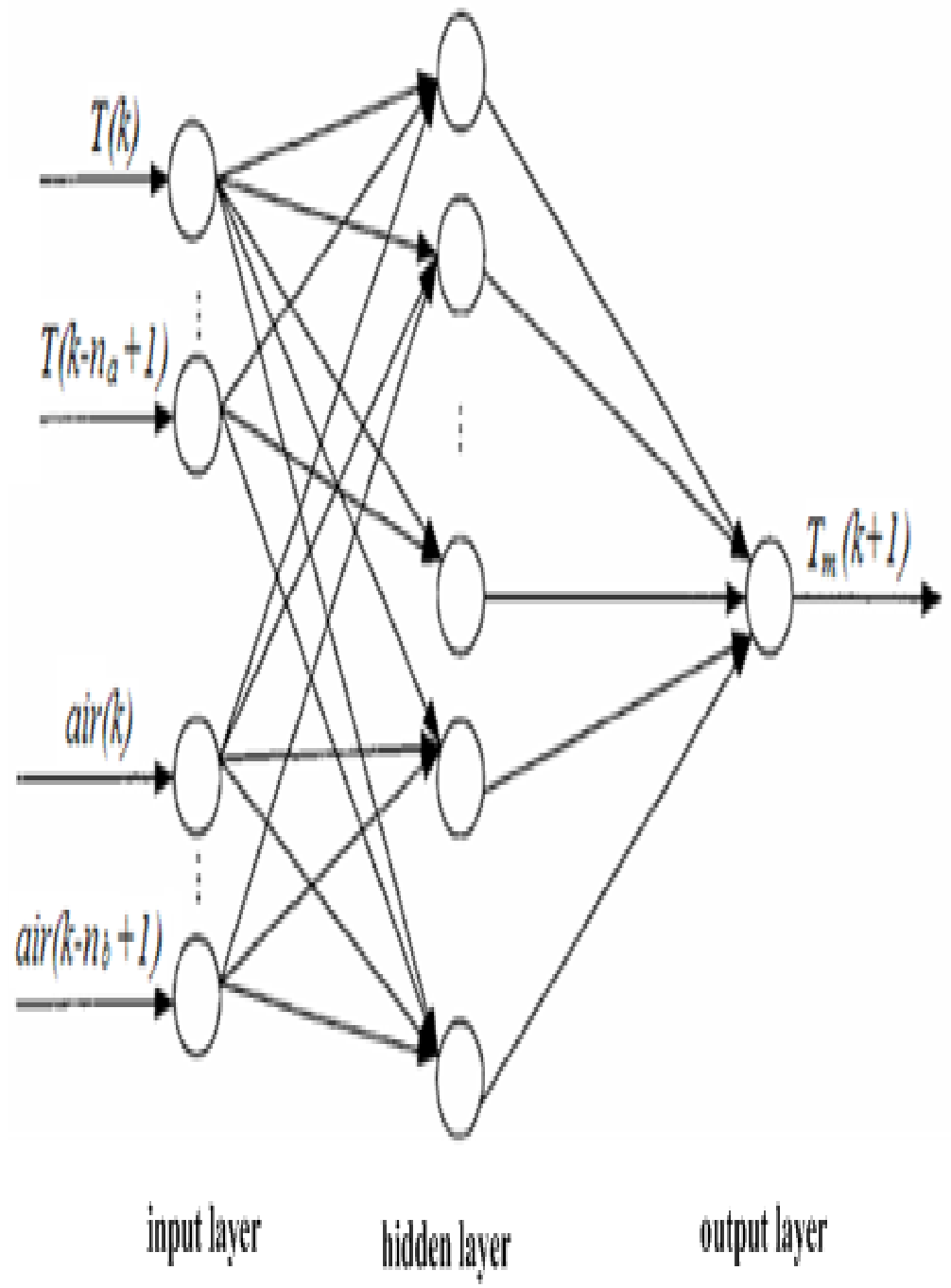

Figure 2 The neural network prediction model

System of temperature and air supply network training samples for $\mathrm{K}$ moment and the moment before.

$$
\begin{array}{clll}
\text { The } & \text { input } \quad \text { for } & \text { network } \\
{\left[T(k), \cdots, T\left(k-n_{a}+1\right), \operatorname{air}(k), \cdots,\right.} & \left.\operatorname{air}\left(k-n_{b}+1\right)\right]^{T} &
\end{array}
$$

The output of the network is $T_{m}(k+1)$

The number of input layer nodes $n_{a}+n_{b}$, The output layer nodes is 1 , The number of hidden layer node is $m_{0}$. 
Since the output has been normalized to the range $[0,1]$. The transfer function of the output layer neurons can be set to S logarithm function. The transfer function model of middle layer neurons are $\mathrm{S}$ tangent function.

\section{Analysis of test results}

To test the feasibility of the control program for the control system of the simulation. At a given temperature of $300 \mathrm{C}$, the simulation results shown in Figure 3. As can be seen from the simulation results, BP neural network predictive control in the nature of the rise time, the transition time are better than traditional PID control, control is better.

In practice, the resistance furnace running state test, the test interval of 2 minutes, the measured data with predicted results shown in Figure 4, the temperature curve of the predicted value and the measured data curves were compared with predicted results difficult to find actual changes in the basic line, even when the signal is too low or too rapid changes.

\section{Conclusion}

Neural network has the massive parallel processing, distributed storage and adaptive, self learning ability of organization. So the neural network is used to realize the algorithm of predictive control model. The simulation results fully demonstrated that the neural network predictive control scheme is successful. It can adapt to the changes of the object, and it showed good control characteristic.

It has strong predictive, robustness, and adaptive. This scheme significantly outperforms the conventional PID control scheme, it has certain guiding significance for practical production.

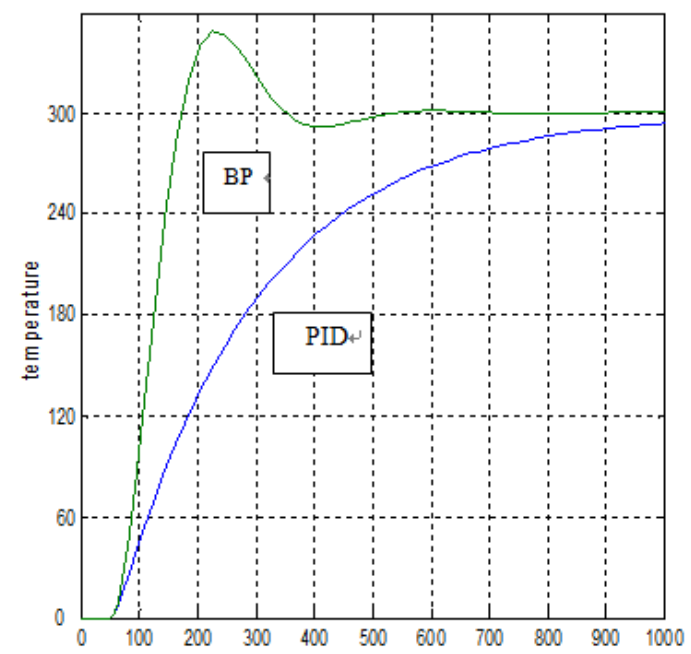

Fig.3 PID control and BP control 


\section{References}

[1] Zhu Jing. Intelligent predictive control and its application[M]. Zhejiang university press. 2002,1-71,105-147.

[2] $\mathrm{Xu}$ Lina.neural network control[M].Beijing Electron Industry Press.2003,18-113.

[3] Li Rui.The application of neural network in nonlinear predictive control[D].Xian university of science and technology.2005.

[4] Li Yuyun, Wang Yongji, Liu Ye.Used in heating furnace of nonlinear neural network predictive controller[J]. The Chinese mechanical engineering. 2001,12(2): 216-219.

[5] Liang Wenbin.Based on the neural network predictive control system research of the robot[D].Yangzhou university.2008.

[6] Chen Bo,Qian Feng,Liu Mandan.A predictive control algorithm based on BP network and its application[J]. Journal of east China university of science and technology.2003,29 (4): 400-404. 